\title{
Management of patients with resistant hypertension: current treatment options
}

This article was published in the following Dove Press journal:

Integrated Blood Pressure Control

21 October 2013

Number of times this article has been viewed

\section{Nilay Kumar' \\ David A Calhoun ${ }^{2}$ \\ Tanja Dudenbostel ${ }^{2}$ \\ 'Department of Medicine, ${ }^{2}$ Division of Cardiovascular Disease, Hypertension and Vascular Biology Program, University of Alabama at Birmingham, Birmingham, AL, USA}

Video abstract

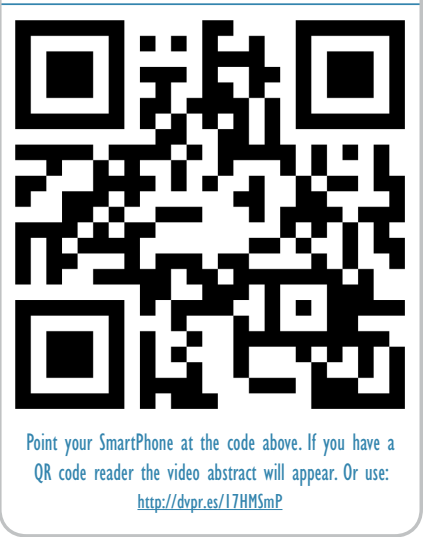

Correspondence: Tanja Dudenbostel UAB Hypertension and Vascular Biology Program, Division of Cardiology,

Department of Medicine,

933 19th Street S, CHSB II5,

Birmingham, AL 35294, USA

$\mathrm{Tel}+\mathrm{I} 205934928$ I

Email tduden@uab.edu
Abstract: Resistant hypertension (RHTN) is an increasingly common clinical problem that is often heterogeneous in etiology, risk factors, and comorbidities. It is defined as uncontrolled blood pressure on optimal doses of three antihypertensive agents, ideally one being a diuretic. The definition also includes controlled hypertension with use of four or more antihypertensive agents. Recent observational studies have advanced the characterization of patients with RHTN. Patients with RHTN have higher rates of cardiovascular events and mortality compared with patients with more easily controlled hypertension. Secondary causes of hypertension, including obstructive sleep apnea, primary aldosteronism, renovascular disease, are common in patients with RHTN and often coexist in the same patient. In addition, RHTN is often complicated by metabolic abnormalities. Patients with RHTN require a thorough evaluation to confirm the diagnosis and optimize treatment, which typically includes a combination of lifestyle adjustments, and pharmacologic and interventional treatment. Combination therapy including a diuretic, a long-acting calcium channel blocker, an angiotensin-converting enzyme inhibitor, a beta blocker, and a mineralocorticoid receptor antagonist where warranted is the classic regimen for patients with treatment-resistant hypertension. Mineralocorticoid receptor antagonists like spironolactone or eplerenone have been shown to be efficacious in patients with RHTN, heart failure, chronic kidney disease, and primary aldosteronism. Novel interventional therapies, including baroreflex activation and renal denervation, have shown that both of these methods may be used to lower blood pressure safely, thereby providing exciting and promising new options to treat RHTN.

Keywords: RHTN, blood pressure, chlorthalidone, hydrochlorothiazide, baroreflex activation, renal denervation, aldosterone, heart failure, renovascular, obstructive sleep apnea

\section{Introduction}

Resistant hypertension (RHTN) is an increasingly common clinical problem ${ }^{1,2}$ that almost always has multiple etiologies, risk factors, and comorbidities. Because of the increasing prevalence of RHTN and its association with increased cardiovascular risk, there has been a growing interest in its epidemiology, pathophysiology, and effective treatment. RHTN is defined as uncontrolled blood pressure (BP) $\geq 140 / 90 \mathrm{mmHg}$ on an optimally dosed three-drug regimen, ideally including a diuretic. The definition also includes patients whose BP is controlled on four or more antihypertensive agents, ie, so-called "controlled resistant hypertension". ${ }^{2}$ In the current article, we review the existing literature on current concepts including lifestyle, pharmacologic, and interventional strategies such as renal sympathetic denervation and baroreflex activation therapy. 


\section{Incidence and prevalence}

The exact incidence and prevalence of RHTN is not known due to the absence of forced titration studies conducted on large hypertensive populations. Data on prevalence of RHTN is derived from cross-sectional studies and post hoc analyses of clinical trials, and is currently estimated at $10 \%-35 \%$ of all patients being treated for hypertension. ${ }^{2}$

The incidence of RHTN was recently investigated in a large retrospective study of 205,750 US adults in the Kaiser Permanente Colorado and Northern California health care systems using patient data collected over a 4-year period. The analysis showed an incidence rate of $1.9 \%$ for RHTN, within a median of 1.5 years of initiation of treatment for hypertension. Patients with RHTN in this cohort were older, more often men, and had a higher prevalence of type 2 diabetes mellitus. Among those taking three or more drugs for at least 4 weeks, the prevalence was found to be $16.2 \% .^{3}$

Data from the National Health and Nutrition Examination Survey (1988-2008) showed that the prevalence of RHTN increased from $5.5 \%$ in $1988-1994$ and $8.5 \%$ in 1999-2004 to $11.8 \%$ in 2005-2008. Patients with RHTN had a greater prevalence of obesity, chronic kidney disease, and Framingham 10-year cardiovascular risk score $>20 \%{ }^{4}$ Data derived from ALLHAT (the Antihypertensive and Lipid Lowering treatment for the prevention of Heart Attack Trial) demonstrated that $27 \%$ of patients required three or more medications to control BP adequately at the end of the study period. ${ }^{5,6}$ In the more recent ACCOMPLISH (Avoiding Cardiovascular events through COMbination therapy in Patients LIving with Systolic Hypertension) study, 25\%-28\% of subjects remained uncontrolled during the course of the study in spite of intensification of treatment. ${ }^{7}$

\section{Prognosis}

Patients with RHTN have higher rates of death, myocardial infarction, congestive heart failure, stroke, and chronic kidney disease compared with those having controlled hypertension. ${ }^{3}$ With the increasing age and prevalence of obesity in the American population, an additional 27 million people are projected to have hypertension by $2030 .{ }^{6}$ As a result, the prevalence of RHTN, its complications, and the associated costs of care are likely to increase significantly. It is therefore imperative for primary care physicians and specialists to identify expeditiously and treat patients with RHTN.

Pseudoresistant hypertension refers to apparent treatment resistance because of poor blood pressure technique, poor adherence, and a possible white coat effect. Pseudoresistance may account for a substantial proportion of apparent RHTN and should be considered if a patient remains resistant to antihypertensive treatment. The true prevalence of pseudoresistance in different populations with RHTN is largely unknown. In an analysis of the Spanish ambulatory blood pressure monitoring registry with 68,045 patients treated for hypertension, $12.2 \%$ were found to have RHTN. ${ }^{8}$ On ambulatory blood pressure monitoring, 37.5\% of RHTN patients were found to have pseudoresistance. Patients with true RHTN had a worse cardiovascular risk profile and greater target organ damage compared with those having pseudoresistance. ${ }^{8}$ In patients with RHTN, lifestyle factors and possible drug-related causes need to be addressed if present. $^{2}$

\section{Secondary causes of resistant hypertension}

Although the exact prevalence of secondary hypertension is not known, patients with RHTN have a greater prevalence of secondary hypertension than the general hypertensive population. ${ }^{2,9}$ RHTN is common in older adults and in individuals with chronic kidney disease, type 2 diabetes mellitus, and obesity. Common causes of secondary hypertension include obstructive sleep apnea, renal artery stenosis (RAS), chronic kidney disease, and primary hyperaldosteronism. Uncommon causes include catecholamine-producing chromaffin cell tumors (pheochromocytoma and paraganglioma), coarctation of the aorta, Cushing's syndrome, secondary hyperparathyroidism, and hyperthyroidism. ${ }^{2}$ We discuss the common causes of secondary hypertension, including obstructive sleep apnea, RAS, and primary hyperaldosteronism, in greater detail below. A comprehensive discussion on chronic kidney disease is beyond the scope of this review.

\section{Obstructive sleep apnea}

Obstructive sleep apnea is a sleep-disordered breathing disease characterized by repeated episodes of apnea-hypopnea during sleep, frequent night-time awakenings, and daytime sleepiness. It is relatively common among American adults and is associated with increased cardiovascular morbidity and mortality. ${ }^{10}$ In the Wisconsin Sleep Cohort Study, the prevalence of obstructive sleep apnea was $9 \%$ for women and $24 \%$ for men. Obesity and male gender were independent predictors of obstructive sleep apnea. ${ }^{11}$ The prevalence of obstructive sleep apnea is particularly high among patients with RHTN. In a study of 71 consecutive individuals referred to a university hypertension clinic, $90 \%$ of men and $77 \%$ 
of women had an apnea-hypopnea index $\geq 5 /$ hour, equivalent to mild obstructive sleep apnea. ${ }^{12}$ In a cross-sectional study of 41 patients with RHTN referred to a university hypertension clinic, $96 \%$ of men and $65 \%$ of women had an apnea-hypopnea index of $\geq 10$, demonstrating a high prevalence of mild obstructive sleep apnea in these patients with RHTN. ${ }^{13}$

The exact mechanisms by which obstructive sleep apnea promotes the development of hypertension are incompletely understood, but evidence indicates that patients with obstructive sleep apnea have increased sympathetic activity and aldosterone levels, as well as a BP increase during progressive stages of sleep. ${ }^{14}$ Recent studies indicate that obstructive sleep apnea is significantly associated with increased aldosterone excretion $^{12}$ and there seems to be a significant correlation between aldosterone levels and the severity of obstructive sleep apnea. ${ }^{15}$ It is hypothesized that chronic parapharyngeal fluid retention, mediated by aldosterone excess and nocturnal rostral fluid shift from the lower extremities, may contribute to worsening airway resistance due to reduced upper airway diameter, thereby adding to the severity of obstructive sleep apnea. ${ }^{16}$ These changes are at least partially attenuated with the use of continuous positive airway pressure (CPAP). ${ }^{14,17}$ Treatment responses are best in patients with high baseline systolic BP, greater severity of obstructive sleep apnea, and better adherence to CPAP. One study showed that in patients with RHTN who used CPAP for $>5.8$ hours per night, there was a greater reduction in 24-hour systolic and diastolic BP as compared with a conventional group and a group using CPAP for $<5.8$ hours per night. ${ }^{18}$ Long-term observational studies have shown a reduction in fatal and nonfatal cardiovascular events with CPAP use in patients with obstructive sleep apnea. ${ }^{10}$

The hypothesis of aldosterone excess worsening severity of obstructive sleep apnea is supported by a prospective study showing a reduction in severity after 8 weeks of treatment with spironolactone in patients with RHTN and obstructive sleep apnea. ${ }^{19}$

\section{Aldosterone and resistant hypertension}

First described by Jerome W Conn in 1955, ${ }^{20}$ primary aldosteronism is characterized by autonomous production of aldosterone by the adrenal glands along with suppressed renin production by the renal juxtaglomerular apparatus. This leads to volume expansion, difficult to control hypertension, metabolic alkalosis, and hypokalemia. While Conn's syndrome consists of an aldosterone-producing adrenal adenoma with hypertension and hypokalemia, the clinical picture of primary hyperaldosteronism has been found to be more diverse. Unilateral/bilateral adrenal (micro)adenomas and/or adrenal hyperplasia are found in patients with primary hyperaldosteronism while other biochemically confirmed cases of primary hyperaldosteronism cases show negative imaging and remain idiopathic. ${ }^{21}$ Hypokalemia is usually a late manifestation of primary hyperaldosteronism, ${ }^{21}$ and normokalemia is quite common among these patients. ${ }^{22}$ Primary hyperaldosteronism is the most common endocrine cause of secondary hypertension to be diagnosed in patients with RHTN. ${ }^{12}$

The prevalence of primary hyperaldosteronism in patients with RHTN is much higher than previously thought. ${ }^{23,24}$ An observational study of 1,616 patients with RHTN indicated a positive screening test for primary hyperaldosteronism in $20.9 \%$ and a confirmed diagnosis of primary hyperaldosteronism in $11.3 \% .^{25}$ In a study of 88 consecutive patients referred to the hypertension clinic at the University of Alabama at Birmingham, $20 \%$ of subjects had confirmed primary hyperaldosteronism with an equal prevalence in African American and Caucasian patients. ${ }^{26}$ Several other prospective studies have reported a similar high prevalence. ${ }^{21,26-28}$

A prominent role of aldosterone excess as a cause of treatment resistance is supported by numerous studies that demonstrate a significant antihypertensive effect when mineralocorticoid receptor antagonists are added, as outlined later in this review.

Recent studies have shown an association of primary hyperaldosteronism, obesity, and obstructive sleep apnea, although the causality of this possible relationship has not been conclusively established. ${ }^{12}$ A prospective population study showed that an increase or decrease in adiposity measured by body mass index leads to similar corresponding changes in $\mathrm{BP}^{29}$ The recently observed high prevalence of primary hyperaldosteronism of approximately $20 \%$ among patients with RHTN coincides with a worldwide epidemic of obesity, suggesting a hitherto unrecognized mechanistic link between the two disease processes. A study of normotensive adults on a high-salt diet demonstrated that body mass index was positively correlated with urinary aldosterone excretion and angiotensin II-mediated aldosterone secretion in normotensive adults. ${ }^{30}$ In 1,125 general hypertensive patients enrolled in PAPY (the Primary Aldosteronism Prevalence in hYpertension study), body mass index was positively correlated with plasma aldosterone concentration independent of age, gender, and sodium intake in patients with essential hypertension but not those with primary hyperaldosteronism. ${ }^{31}$ Weight reduction leads to a decrease in aldosterone levels, 
suggesting that adipose tissue may play a role in generalized activation of the renin-angiotensin-aldosterone system. ${ }^{32}$ As stated above, the plasma aldosterone concentration correlates positively with the severity of obstructive sleep apnea, and spironolactone seems to attenuate that effect. The exact mechanisms whereby adipocytes may promote hyperaldosteronism are not completely understood. Current evidence indicates that obesity may promote a generalized activation of the renin-angiotensin-aldosterone system since adipocytes possess all the components of this pathway. ${ }^{33}$ In a recent experimental study, ${ }^{34}$ the mineralocorticoid/aldosteroneproducing activity of human adrenocortical cells increased seven-fold when incubated for 24 hours with secretory products from adipocytes. This effect was independent of angiotensin II and persisted in the presence of the angiotensin (AT)1 receptor antagonist, valsartan, suggesting that an adipocyte-derived secretagogue other than angiotensin II was responsible for this effect. In another study by the same group, adipocyte-derived factors/adipokines increased the sensitivity of human adrenocortical cells to angiotensin II stimulation. This effect was mediated by extracellular signalregulated kinase (ERK) 1/2 mitogen activated protein (MAP) kinase-dependent stimulation of steroidogenic acute regulatory protein, and blockade of ERK 1/2 MAP kinase abolished adipokine-induced aldosterone secretion. ${ }^{35}$ These data suggest that adipocyte-derived factors upregulate aldosterone production, directly or indirectly, and elevated aldosterone levels may be one factor in adverse cardiovascular outcomes associated with obesity.

\section{Renovascular disease}

\section{Atherosclerotic renal artery stenosis}

Another cause of secondary hypertension is renovascular hypertension caused by a stenotic lesion within the renal artery. ${ }^{36,37}$ As with other secondary causes of hypertension, RAS should be suspected when BP control is resistant to medical treatment ${ }^{2}$ or if a patient presents with a history of controlled hypertension that suddenly becomes resistant. In some patients with RAS, renal insufficiency or a sudden decrease in renal function, proteinuria, or even nephrotic syndrome may be detected..$^{38}$

More than $90 \%$ of RAS is atherosclerotic in origin. The likelihood of atherosclerotic renal artery stenosis (ARAS) is increased in patients with classic cardiovascular risk factors like age, smoking, dyslipidemia, and diabetes. ${ }^{37}$ While the prevalence of ARAS in the general population of patients older than 65 years has been found to be $7 \%,{ }^{7}$ studies of patients with RHTN demonstrate a higher prevalence of previously unrecognized renovascular disease, particularly in older high-risk patient groups. ${ }^{39,40}$ ARAS is a common finding in hypertensive patients undergoing cardiac catheterization. In a meta-analysis of 27 studies with a total of 30,092 patients undergoing diagnostic coronary angiography for suspected coronary artery disease, the prevalence of ARAS $>50 \%$ averaged $8 \%$ with a range from $3.1 \%-22.9 \%$. ${ }^{41}$ Of all patients with ARAS, significant bilateral ARAS was found in $20.3 \% .{ }^{41}$ The prognosis of ARAS has been investigated in a group of patients undergoing coronary angiography, ${ }^{42}$ and the study demonstrated that the presence of ARAS is independently associated with mortality, with the lowest survival rate in patients with bilateral ARAS. ${ }^{42}$ In a study with a total of 3,987 patients who underwent abdominal aortography immediately following coronary angiography a survival analysis was done. The four-year unadjusted survival for patients with and without significant ARAS was $57 \%$ and $89 \%$, respectively $(P<0.001)$. In a multivariate model, the presence of ARAS conferred a hazard ratio of 2.01 (95\% confidence interval 1.51-2.67, $P<$ 0.001). ARAS is a strong independent predictor of mortality and increasing severity of ARAS has an incremental effect on probability of survival. ${ }^{42}$

Unilateral or bilateral ARAS should be suspected in patients with a history of flash or recurrent pulmonary edema, especially when echocardiography indicates preserved systolic ejection fraction. It was recently proposed to name this clinical entity after the late Thomas G Pickering ${ }^{41}$ who reported a series of 11 hypertensive patients with bilateral ARAS presenting with a history of multiple episodes of pulmonary edema in $1988,{ }^{43}$ which inspired further research in this field. ${ }^{41}$

The mechanism by which RAS leads to hypertension and recurrent pulmonary edema, the so-called Pickering syndrome, is thought to be defective natriuresis, an acute increase in hemodynamic burden, exacerbation of diastolic dysfunction, and failure of the pulmonary capillary blood-gas barrier. ${ }^{41}$ However, the means by which nonacute ARAS leads to maintenance and progression of hypertension as well as accelerated end-organ damage is not well understood. ${ }^{36}$

Imaging of the renal arteries has been found to be a reliable tool with high sensitivity and specificity depending on the imaging technique. Renal artery ultrasound with duplex is a useful first-line screening method. Computed tomographic angiography and magnetic resonance angiography with gadolinium have higher sensitivity and specificity than renal artery ultrasound with duplex to visualize stenosis. ${ }^{44}$ Although digital subtraction angiography is an invasive test, it is considered the reference standard and allows simultane- 
ous revascularization with angioplasty and stent placement in addition to its diagnostic utility. ${ }^{44}$

In patients with recurrent flash pulmonary edema or Pickering syndrome, the evidence indicates that percutaneous revascularization is the therapy of choice and stenting seems to be superior over balloon angioplasty alone. ${ }^{45-47}$

In patients with unilateral nonacute $\mathrm{ARAS},{ }^{2}$ the optimal strategy is less clear, and both medical and revascularization strategies should be considered to control BP, preserve renal function, and reduce the risk of cardiovascular disease and mortality. Studies that compared these treatment options have been largely inconclusive. To date, three randomized trials of stenting versus medical therapy have been published, ie, the DRASTIC (Dutch Renal Artery STenosis Intervention Cooperative) trial, ${ }^{48}$ the STAR (Stent Placement in Patients with Atherosclerotic Renal Artery Stenosis and Impaired Renal Function) study, ${ }^{49}$ and the recently published multicenter, randomized, nonblinded ASTRAL (Angioplasty and STenting for Renal Artery Lesions) trial that assigned 806 patients with ARAS to undergo revascularization in addition to receiving medical therapy or to receive medical therapy alone. ${ }^{50}$ All three trials concluded that revascularization with stent placement had no clinical benefit over medical treatment and found a significant risk of procedure-related complications. ${ }^{37}$ However, there has been some criticism related to sample size, patient selection, methodology, center selection, lesion assessment, and verification by a core laboratory. ${ }^{51} \mathrm{On}$ the one hand, the above-mentioned randomized trials did not show convincing benefit in regard to improvement in renal function or BP control by revascularization of RAS; on the other hand, these trials had major flaws in design. ${ }^{52}$

The large-scale, randomized CORAL (Cardiovascular Outcomes in Renal Atherosclerotic Lesions) trial ${ }^{45}$ recently completed enrollment and randomization of more than 900 patients with $\geq 60 \%$ stenosis to either medical treatment alone or optimal medical therapy plus renal artery stenting. Results from CORAL are pending, but should provide guidance as to whether renal artery stenting is beneficial in the patient population studied or not. Until then, revascularization remains controversial due to the lack of well controlled, well designed, prospective, randomized trials, and treatment must be individualized by case. However, in patients with RHTN, revascularization with stenting for ARAS is generally recommended. ${ }^{53}$ Currently accepted indications for endovascular management in significant RAS $>70 \%$ by renal artery ultrasound with duplex or digital subtraction angiography are a progressive or acute decline in renal function with or without introduction of an angiotensin-converting enzyme (ACE) inhibitor, true RHTN, solitary kidney, recurrent flash pulmonary edema, or other cardiac disturbance syndromes. ${ }^{45,52}$

Medical therapy for ARAS should include more than one class of antihypertensive agent. Treatment should include an ACE inhibitor or angiotensin II receptor blocker (ARB), a calcium channel blocker, and if necessary a beta-blocker. ${ }^{53}$ While many physicians retain reservations about using reninangiotensin blocking drugs in patients with bilateral ARAS, in a prospective observational study, RAAS blockade was well tolerated in $78.3 \%$ of patients with bilateral $>60 \%$ ARAS ${ }^{54}$ However, use of an ACE inhibitor should be evaluated on an individual basis, and serum creatinine/glomerular filtration rate and potassium levels should be monitored closely when treatment is initiated. ${ }^{55}$ Additional to BP control treatment aiming at reduction of cardiovascular risk factors like lipid-lowering drugs, antiplatelet agents, treatment of diabetes, and sequelae of chronic kidney disease according to current guidelines, is recommended. ${ }^{2}$

\section{Renal artery fibromuscular dysplasia}

Less than $10 \%$ of renal artery lesions are caused by renal artery fibromuscular dysplasia (FMD), ${ }^{36}$ a medial fibroplasia with different subtypes causing so-called string of beads where the bead is larger than the normal caliber of the artery. The prevalence of FMD in patients with RHTN is unknown. Data from the US Registry for FMD showed a median number of two antihypertensive medications with an interquartile range of 1-3, suggestive of RHTN in some patients with FMD. ${ }^{56}$ In patients with new-onset RHTN or RHTN, FMD should be considered as a secondary cause. FMD is predominantly found in women younger than 50 years of age, but is also found in the elderly. ${ }^{57}$ Recent data from the US Registry demonstrated a mean age at first symptoms of $47.2 \pm 14.6$ (range 5-83) years and mean age at first clinical diagnosis of $51.9 \pm 13.4$ years. ${ }^{56}$ While data from the US showed that approximately nine of ten patients diagnosed with renal artery FMD are female and white, investigators from France recently reported that in 337 patients who were referred for hypertension and renal artery FMD, 17\%-31\% were male. ${ }^{56}$ Because renal artery FMD is now being recognized in elderly persons, it is not uncommon to encounter patients with both atherosclerosis and FMD. ${ }^{57-60}$ Data from the US Registry demonstrate that among 337 patients with renal or mesenteric imaging and a documented physical examination for epigastric or flank bruits, 262 (77.7\%) had FMD. The sensitivity of an epigastric or flank bruit for identifying renal or mesenteric FMD was 63/262 (24.0\%) with a specificity of $70 / 75(93.3 \%) .{ }^{56}$ Thus, the presence of a bruit in a young 
or middle-aged patient should raise suspicion and warrants further investigation. However, the finding of an abdominal bruit has a high specificity for ARAS and FMD,${ }^{61}$ but is not found in every patient and the lack of an abdominal bruit does not rule out ARAS or FMD.

\section{Treatment Lifestyle \\ Low-sodium diet}

Dietary sodium reduction has been shown to be effective in lowering BP, especially in African Americans and the elderly. ${ }^{62}$ Both observational studies and clinical trials have indicated that increased sodium intake is associated with increased BP, and it is currently recommended that sodium intake should be less than $1,500 \mathrm{mg}$ /day in hypertensive patients. In a recent, prospective, crossover study of subjects with RHTN, a low-sodium diet was compared with a high-salt diet. ${ }^{63}$ Twelve subjects with resistant hypertension entered into a randomized crossover evaluation of low $(50 \mathrm{mmol} / 24$ hours $\times 7$ days $)$ and high $(250 \mathrm{mmol} / 24$ hours $\times 7$ days $)$ sodium diets, separated by a 2-week washout period. ${ }^{64}$ Mean urinary sodium excretion was 46 versus $252 \mathrm{mmol} / 24$ hours during low-sodium versus high-sodium intake. When compared with a high sodium intake, lower sodium intake was associated with a marked decrease in BP of 22.7/9.1 mmHg. Remarkably, BP reduction seen with sodium restriction is equivalent to the effect of almost two antihypertensive agents, thus emphasizing the importance of including a low-sodium diet in the treatment plan for RHTN.

\section{High-fiber, low-fat diet}

A diet rich in fruit and vegetables, potassium, magnesium, calcium, and low-fat dairy products and low in total saturated fats (ie, the Dietary Approaches to Stop Hypertension [DASH] diet) should be recommended for all hypertensive patients, including all patients with RHTN. In a prospective, randomized clinical study, the effect of a control diet (low in fruit, vegetables, and dairy products, with a fat content typical of the average diet in the US), a diet rich in fruit and vegetables, or a "combination" diet rich in fruit, vegetables, and low-fat dairy products and with reduced saturated and total fat was assessed in 459 healthy and hypertensive adults with a mean BP of 131.3/84.7 mmHg at baseline. ${ }^{65}$ Sodium intake and body weight were maintained at constant levels. The greatest effect was seen in subjects with hypertension. Among those subjects, the combination diet reduced BP by $11.4 / 5.5 \mathrm{mmHg}$ more than the control diet. Among the subjects without hypertension, the corresponding reductions were 3.5/2.1 mmHg. This demonstrates that a diet rich in fruit, vegetables, and low-fat dairy products with reduced saturated and total fat offers an additional nutritional approach to prevent and treat hypertension.

\section{Weight loss}

Obesity has been shown to be associated with RHTN and an increased number of antihypertensive agents. ${ }^{66}$ Consistent with this observation, weight loss studies have been shown to be effective in BP-lowering. A review of weight loss studies demonstrated that an approximately $20 \mathrm{lb}$ weight loss is associated with an average $6 / 4.6 \mathrm{mmHg} \mathrm{BP}$ reduction. ${ }^{67}$

\section{Physical activity}

In a meta-analysis that included randomized studies of normotensive and hypertensive patients, regular aerobic exercise produced average BP reductions of $4 / 3 \mathrm{mmHg} .{ }^{68}$ In a small group of African American men with RHTN, 16 weeks of stationary cycling three times a week reduced BP by $7 / 5$ mmHg. ${ }^{69}$ Reductions in diastolic BP were maintained after 32 weeks of exercise, even with withdrawal of some antihypertensive medications.

A recent randomized, controlled trial examined the cardiovascular effects of aerobic exercise in 50 subjects with RHTN randomly assigned to participate or not to participate in an 8-12-week treadmill exercise program. ${ }^{70}$ Exercise significantly decreased ambulatory BP by a mean $6 / 3$ (standard deviation 12/7) $\mathrm{mmHg}(P=0.03)$. Based on these observed BP-lowering effects, endurance exercise should be included in the therapeutic approach for patients with RHTN.

\section{Pharmacologic therapy}

One of the most challenging problems in treating RHTN is the appropriate antihypertensive drug combination. Recently, a large, retrospective cohort study described use of antihypertensive agents in patients with RHTN. ${ }^{71}$ The most frequently prescribed antihypertensive classes were an ACE inhibitor and/or ARB in $96.2 \%$, a diuretic in $93.2 \%$, a calcium channel blocker in $83.6 \%$, and a beta-blocker in $80.0 \%$ of patients. Only $3.0 \%$ and $5.9 \%$ of patients were on chlorthalidone or a mineralocorticoid receptor antagonist, respectively, both of which are evidence-based and recommended antihypertensive agents. The underuse of chlorthalidone is particularly significant, given its antihypertensive efficacy compared with other thiazides, longer duration of action, and better cardiovascular risk profile. Moreover, minimally efficacious combinations, such as 
an $\mathrm{ACE}$ inhibitor and an $\mathrm{ARB}$, were prescribed in a total of $15.6 \%$.

A recent, large, retrospective cohort study demonstrated that prescription of different classes of antihypertensive drugs decreased one year after the diagnosis of RHTN. ${ }^{72}$ Among the most prominent reductions in antihypertensive drug prescriptions was a $12 \%$ decline in the use of diuretic agents. The authors found that lack of treatment intensification but not medication adherence was significantly associated with reduced BP control.

One cause of RHTN is significant volume overload, and diuretic therapy is essential in this patient group. Recently, chlorthalidone and mineralocorticoid receptor antagonists have been emphasized as a treatment strategy for patients with RHTN. ${ }^{73,74}$

\section{Diuretics}

A prospective study evaluated the utility of hemodynamic parameters in the selection and titration of antihypertensive agents. The investigators found that patients with RHTN often had occult volume expansion underlying their treatment resistance, and BP control was improved primarily through forced titration of diuretics. ${ }^{75}$

These studies showed that patients with RHTN frequently have increased volume load contributing to treatment resistance and that treatment with a diuretic agent is essential for effective BP reduction. Thiazide diuretics are effective antihypertensive agents in the majority of patients. Recently, several landmark trials have demonstrated that chlorthalidone, a thiazide-like long-acting diuretic, reduces BP and, more important, the risk of stroke and other cardiovascular endpoints. In a randomized, single-blind, 8-week, active-treatment, crossover study comparing chlorthalidone $12.5 \mathrm{mg}$ once daily (force-titrated to $25 \mathrm{mg}$ once daily) and hydrochlorothiazide $25 \mathrm{mg}$ once daily (force-titrated to $50 \mathrm{mg}$ once daily) in untreated uncontrolled hypertensive patients, 24-hour ambulatory BP indicated a greater reduction from baseline with chlorthalidone $25 \mathrm{mg}$ once daily than with hydrochlorothiazide $50 \mathrm{mg}$ once daily (24-hour mean of $-12.4 \pm 1.8 \mathrm{mmHg}$ versus $-7.4 \pm 1.7 \mathrm{mmHg} ; P=0.054$; night-time mean of $-13.5 \pm 1.9 \mathrm{mmHg}$ versus $-6.4 \pm 1.8 \mathrm{mmHg} ; P=0.009) .{ }^{76}$ Based on the beneficial effects on cardiovascular outcomes and its higher potency, chlorthalidone should be preferentially used in patients with RHTN and a glomerular filtration rate $>30 \mathrm{~mL} / \mathrm{min} / 1.73 \mathrm{~m}^{2} .{ }^{.7}$

In patients with chronic kidney disease stage 4 or 5 (glomerular filtration rate $<30 \mathrm{~mL} / \mathrm{min} / 1.73 \mathrm{~m}^{2}$ ), loop diuretics may be necessary for effective volume and BP control.
Furosemide is a short-acting loop diuretic and requires twicedaily dosing, with the second dose after 6-8 hours. Alternatively, torsemide, a longer-acting loop diuretic, can be used once daily to simplify the regimen. In patients with congestive heart failure, an entity common in patients with RHTN, one study demonstrated that torsemide-treated patients were less likely to be readmitted for congestive heart failure and for all cardiovascular causes, and were less fatigued compared with those treated with furosemide. ${ }^{78}$

\section{Mineralocorticoid receptor antagonists}

Mineralocorticoid receptor antagonists have been shown to be effective fourth-line BP-lowering agents in African American and Caucasian patients with and without primary hyperaldosteronism. ${ }^{79-81}$ One recent prospective study demonstrated the BP-lowering effect of spironolactone $25 \mathrm{mg}$ once daily in patients with RHTN on an average of four medications including an ACE inhibitor or ARB and a diuretic. After 6 months, BP was reduced by an average of $25 / 12 \mathrm{mmHg} .{ }^{79}$ Similar data were found in ASCOT (the Anglo-Scandinavian Cardiac Outcome Trial), in which 1,411 participants had a significant BP reduction of 22/10 mmHg after a mean 1.3 years of follow-up when spironolactone $25 \mathrm{mg}$ daily was administered as a fourthline therapy. ${ }^{82}$ More recently, a study in 175 patients with RHTN prospectively determined the BP reduction by adding spironolactone $25-100 \mathrm{mg}$ daily to an existing standard regimen. ${ }^{83}$ After a median 7 months of follow-up, there were mean BP reductions of $16 / 9 \mathrm{mmHg}$.

A growing body of evidence links aldosterone to the development and progression of several cardiovascular disease processes, including congestive heart failure, independent of BP levels. Recently, a prospective study in more than 250 patients with RHTN demonstrated that even patients with normal or low aldosterone levels had evidence of greater intravascular fluid retention, as shown by higher brain natriuretic peptide and atrial natriuretic peptide levels in comparison with controls. ${ }^{84}$ In addition, structural and hemodynamic parameters, including left ventricular hypertrophy and ventricular volumes, were determined at 3-month and 6-month follow-up in patients prospectively treated with spironolactone $25 \mathrm{mg}$ once daily, and if indicated, titrated to $50 \mathrm{mg}$ once daily. Significant rapid decreases in BP and left ventricular hypertrophy were seen in patients with and without primary hyperaldosteronism at just 3 months, with effects persisting to 6 months. In patients with primary hyperaldosteronism, in addition to a significant decrease in BP and left ventricular hypertrophy, a significant reduction 
of right and left ventricular volumes and brain natriuretic peptide was noted, supporting a relative volume overload state that may underlie treatment resistance in patients with aldosterone excess. $^{84}$

RALES (the Randomized ALdactone Evaluation Study) indicated that addition of spironolactone $25 \mathrm{mg}$ once daily to a regimen that included an ACE inhibitor in most patients with congestive heart failure significantly improved survival by $30 \% .{ }^{85}$ Similar results were found in EPHESUS (the Eplerenone Post-Acute Myocardial Infarction Heart Failure Efficacy and Survival Study) when eplerenone was added to an ACE inhibitor or ARB, and a beta-blocker, survival was improved by $31 \%$ in patients with left ventricular dysfunction after acute myocardial infarction. ${ }^{86}$ In showing benefit by directly blocking the mineralocorticoid receptor, these intervention studies indicate that aldosterone may be contributing directly to development and progression of cardiovascular disease independent of BP.

Although spironolactone was generally well tolerated in these studies, breast tenderness occurred in approximately $10 \%$ of male patients. This may be avoided by use of eplerenone, a selective mineralocorticoid receptor antagonist that has been shown to lower BP effectively and may also have beneficial effects on the progression of renal disease. In an open-label study, patients who received 50-100 mg of eplerenone daily were followed for 12 weeks. ${ }^{87} \mathrm{Clinic}$ and 24-hour ambulatory $\mathrm{BP}$ was reduced by $18 / 8 \mathrm{mmHg}$ and $12 / 6 \mathrm{mmHg}$, respectively, and the BP-lowering effect did not appear to be influenced by underlying aldosterone status.

Amiloride is, like mineralocorticoid receptor antagonists, a potassium-sparing diuretic that indirectly acts as a direct inhibitor of the aldosterone-regulated epithelial sodium channel in the distal nephron. In a prospective, randomized, placebo-controlled, double-blind clinical study in 98 African American patients with uncontrolled low-renin hypertension despite treatment that included a diuretic and a calcium channel blocker, treatment with either amiloride or spironolactone provided a comparable additional reduction in BP, and synergistic effects were seen with the combination of both agents. ${ }^{88}$ In a substudy, plasma endothelin-1 levels were observed to decrease significantly after 3 weeks of treatment in the spironolactone group, suggesting a broader effect than inhibition of the epithelial sodium channel alone.

Studies using amiloride or spironolactone or the combination of both diuretics ${ }^{88}$ have demonstrated that potassiumsparing diuretics are, in general, safe agents, with development of hyperkalemia being uncommon despite concurrent use of an ACE inhibitor or ARB and a beta-blocker. Potassium levels must be monitored closely, however, especially in elderly patients with chronic kidney disease and/or diabetes who are at higher risk of developing hyperkalemia. In these patients, spironolactone therapy should be commenced at a lower dose (12.5 mg daily), nonsteroidal anti-inflammatory drugs and potassium supplements should be discontinued, and renal function should be closely monitored.

\section{Combination therapy}

An important principle of antihypertensive therapy in resistant hypertensive patients is that greater BP reduction is achieved by combining three drugs from different classes at maximum dose, with one ideally being a diuretic. Additional to a long-acting dihydropyridine calcium channel blocker like amlodipine, other antihypertensive agents usually include an ACE inhibitor or an ARB ${ }^{89}$ However, there are little data assessing the efficacy of specific combinations of three or more drugs in patients with RHTN. It has been suggested that the combined use of an ACE inhibitor and ARB or a dihydropyridine and nondihydropyridine calcium channel blocker provides significant additional antihypertensive benefit in patients with essential hypertension. In ONTARGET (the ONgoing Telmisartan Alone and in combination with Ramipril Global Endpoint Trial), the combination of an ACE inhibitor and an ARB was assessed and the study demonstrated that subjects $\geq 55$ years with established atherosclerotic vascular disease or with type 2 diabetes mellitus and end-organ damage had a greater reduction of proteinuria with the combination of an ACE inhibitor and ARB than with monotherapy. ${ }^{89}$ However, overall, the combination of an ACE inhibitor and ARB worsened major renal outcomes and, despite reductions in BP, no significant benefit was seen in the primary composite outcome of death from cardiovascular causes, myocardial infarction, stroke, or hospitalizations for congestive heart failure, even in high-risk patients. ${ }^{90}$ Recent studies as discussed above indicate that mineralocorticoid receptor antagonist as a fourth or fifth addon to antihypertensive agent may be beneficial. ${ }^{79-86}$

In the setting of coronary artery disease or congestive heart failure, combined alpha-beta blockade is recommended to improve cardiovascular outcomes for these patients, and so-called vasodilating beta-blockers may be beneficial in patients with RHTN as well. However, prospective headto-head comparisons of large cohorts of diverse patients with RHTN are lacking. Ultimately, the clinician needs to individualize the drug regimen for patients with RHTN and consider prior efficacy and comorbidities, adverse effects, and financial restraints. 


\section{Novel device therapies \\ for resistant hypertension}

Baroreflex activation therapy

with carotid stimulation

There is an increasing body of research that implicates the carotid baroreceptors as being activated by pressure that leads to compensatory adjustments in the sympathetic and parasympathetic nervous system involved in regulation of BP control. ${ }^{91}$ Electrical stimulation of carotid baroreceptors has been shown to elicit a depressor response through sympathetic inhibition. The Rheos ${ }^{\circledR}$ Hypertension Therapy System ${ }^{\mathrm{TM}}$ (Rheos, Minneapolis, MN, USA) is an implantable device consisting of a pulse generator and two electrode leads that are placed bilaterally to chronically activate carotid artery baroreceptors. ${ }^{92}$ In the Rheos Pivotal Trial, the baroreflex activation therapy device of the first generation (CVRx, Rheos) was evaluated. In this double-blind, randomized, placebo-controlled trial that enrolled 322 subjects with RHTN, ${ }^{93}$ the Rheos device was implanted in 265 subjects who were subsequently randomized to immediate baroreflex activation therapy for the first 6 months or deferred baroreflex activation therapy after 6 months. Primary outcome measures, including significant reduction of office systolic BP at 6 months, sustained response through 12 months, and device safety were assessed. Treatment failed to show a significant reduction of 6-month BP and procedural safety. However, it did show significant benefit for 12-month efficacy and baroreflex activation therapy-related and device-related safety. Interestingly, those randomized to having the pulse generator activated within one month showed a $40 \%$ drop in "serious adverse events for hypertensive urgency" at 6 months. By 12 months, all patients had achieved systolic BP reductions of up to $35 \mathrm{mmHg}$ compared with baseline, and systolic BP had fallen to $\leq 140 \mathrm{mmHg}$ in more than $50 \%$ of the study population. In detail, changes at 6 and 12 months from pre-implant yielded a decrease in systolic BP of $26 \pm 30 \mathrm{mmHg}$ for the group with implant activated and $17 \pm 29 \mathrm{mmHg}$ for the group without implant activated $(P=0.03)$ at 6 months and a decrease of $35 \pm 28 \mathrm{mmHg}$ for group A (BAT for the first 6 months) and $33 \pm 30 \mathrm{mmHg}$ for group B (delayed BAT initiation following the 6-month visit) $(P=0.57)$ at 12 months. At 12 months, the systolic BP of $81 \%$ of subjects had dropped at least $10 \mathrm{mmHg}$ from pre-implant. This responder group experienced an average systolic BP drop of $44 \mathrm{mmHg}$, and $63 \%$ of these subjects reached a systolic BP of $\leq 140 \mathrm{mmHg}$.

The trial failed to show procedural safety due to carotid sinus lead placement and involved transient (4.4\%) or permanent nerve injury ( $4.8 \%$ with some residual effect) that occurred at the time of implant. Additionally, there were 4.8\% of subjects with a general surgical complication and 2.6\% with either a respiratory complaint or a wound complication following implant. The majority $(76 \%)$ of procedurerelated adverse events resolved completely and there were no procedure-related deaths. ${ }^{94}$

Barostim $n e o^{\mathrm{TM}}$ (CVRx; Minneapolis, MN, USA) is the second-generation device that has been designed to deliver baroreflex activation therapy with a simpler implantable device and procedure. In a single-arm, open-label, nonrandomized study, 30 subjects with RHTN were enrolled from seven centers in Europe and Canada to evaluate safety, efficacy, and change from baseline in systolic BP at 6 months. There was a significant decrease of BP by $26 \pm 4.4 / 12.4 \pm 2.5 \mathrm{mmHg}$ at 6 months, consistent with studies of the first-generation system. Although encouraging, results from larger studies are needed to determine whether the Barostim $n e o^{\mathrm{TM}}$ system is a safe and efficient device. For this reason, the Barostim neo ${ }^{\mathrm{TM}}$ study is currently enrolling approximately 300 subjects with RHTN at several clinical sites in the US. In this randomized, open-label parallel-assigned treatment trial, the primary outcome measures are safety and efficacy. Secondary outcome measures are a significant reduction of office systolic BP and 24-hour ambulatory systolic BP between baseline and 12 months post-activation.

\section{Renal denervation therapy}

A novel investigational technique for the treatment of RHTN is renal sympathetic denervation (RDN). This therapeutic strategy uses low energy radiofrequency delivered via a percutaneously inserted catheter for the bilateral disruption of afferent and efferent sympathetic nerves in the adventitia of renal arteries. ${ }^{95,96}$ SYMPLICITY HTN-1 was a multicenter, proof-of-principle, cohort study to demonstrate the feasibility, efficacy, and safety of catheter-based therapeutic RDN in patients with RHTN. In this study, 45 patients with RHTN and a mean BP of 177/101 mmHg on a mean of 4.7 antihypertensive medications underwent RDN treatment. BP reduction was 21/11 $\mathrm{mmHg}$ and 27/17 $\mathrm{mmHg}$ at 6 and 12 months, respectively. Cardiac magnetic resonance imaging 12 months after the procedure showed a decrease in left ventricular mass of $9 \% .{ }^{97} \mathrm{~A}$ follow-up study at 24 months of SYMPLICITY HTN-1 showed a BP reduction of $32 / 14 \mathrm{mmHg}$, thus demonstrating the long-term antihypertensive effect of RDN. While the results of SYMPLICITY HTN-1 were encouraging, the study did not include a control group and investigators and subjects were not blinded in the study. A placebo effect could therefore not be excluded. The 
absence of a control group also made the study prone to both the Hawthorne effect as well as regression to the mean. The subsequent SYMPLICITY HTN-2 trial was a multicenter, prospective, randomized, controlled, open-label, follow-up trial that enrolled a total of 106 patients randomized to RDN and medical management or medical management alone. The primary efficacy endpoint for the study was the change in office BP at 6 months. Office BP at 6 months in the RDN group was reduced by $32 / 12 \mathrm{mmHg}$ while there was no change in the control group. In a subset of patients, 24-hour ambulatory BP measurement showed a significant reduction of $11 / 7 \mathrm{mmHg} 6$ months after treatment. ${ }^{96}$ One-year follow-up of the SYMPLICITY HTN-2 cohort assigned to RDN showed that the mean reduction in systolic BP at 12 months was similar to that observed at 6 months (28 versus $32 \mathrm{mmHg}$, $P=0.16)$. A crossover to the RDN group was permitted in control patients. ${ }^{98}$ In 35 patients who underwent crossover to the RDN group, a significant BP reduction of $24 / 8 \mathrm{mmHg}$ was observed at 6 months, ${ }^{98}$ and the reduction in BP was similar to that in the original RDN cohort at 6 months. Limitations of SYMPLICITY HTN-2, including its nonblinded design and missing sham procedure in the control group, are currently addressed by SYMPLICITY HTN-3, which is a regulatory study designed as a multicenter, prospective, single-blind, randomized, controlled study. Actively treated patients will undergo an RDN procedure and medical therapy, whereas the control group will undergo renal angiography alone as a sham procedure, with identical post-procedure care to maintain subject blinding. The primary outcome measure is change in office systolic BP from baseline to 6 months post-randomization and the primary effectiveness outcome measure is the incidence of major adverse events through one month post-randomization, including RAS de novo or progression of pre-existing RAS measured at 6 months. The secondary outcome measure is change in average 24-hour systolic BP by ambulatory BP monitoring. ${ }^{99}$

In SYMPLICITY HTN-1 and SYMPLICITY HTN-2, renal angiography and magnetic resonance angiography performed in a subset of patients at 2-4 weeks post procedure showed that there was no delayed RAS in response to RDN. ${ }^{95,96}$ One renal artery dissection and one femoral pseudoaneurysm occurred in SYMPLICITY HTN-1, while one femoral pseudoaneurysm occurred in SYMPLICITY HTN-2. All complications were successfully treated, thus establishing the safety of the RDN procedure. ${ }^{95,96}$ Long-term outcome data are currently awaited from the two SYMPLICITY cohorts.

The promising results of the SYMPLICITY studies have led to the development of novel four-electrode catheters that deliver radiofrequency simultaneously and are designed to reduce procedure time significantly as compared with a single electrode at the tip of the catheter. Studies investigating the efficacy and safety of four-electrode radiofrequency catheters provided by Medtronic and St Jude are underway, the latter investigating cardiovascular outcomes. Several studies are currently being conducted to investigate the effects of RDN additional to its BP-lowering effects on various cardiovascular risk factors, secondary causes, and comorbidities. RDN may be a potential option in patients who have exhausted lifestyle modifications, thorough workup for secondary causes, and pharmacologic treatment to control hypertension.

\section{Conclusion}

RHTN is a common clinical problem encountered by internists and specialists. Patients with RHTN should be screened for reversible causes of hypertension, such as RAS, primary aldosteronism, and obstructive sleep apnea. Patients should be counseled about potentially modifiable lifestyle factors, such as sodium intake, weight loss, diet, and exercise. Combination therapy with different antihypertensive classes is crucial in the effective treatment of RHTN. This should ideally include a thiazide-like diuretic like chlorthalidone because of its longer duration of action and better antihypertensive efficacy. The role of aldosterone excess in the pathogenesis of RHTN is being increasingly recognized, and addition of a mineralocorticoid receptor antagonist as a fourth-line antihypertensive agent is recommended. Clinical trials evaluating the safety and efficacy of novel therapies including baroreflex activation therapy and $\mathrm{RDN}$ are currently underway, with favorable preliminary data. These techniques have the potential to add an exciting new dimension to the existing repertoire of antihypertensive therapies.

\section{Disclosure}

The authors report no conflicts of interest in this work.

\section{References}

1. Lim SS, Vos T, Flaxman AD, et al. A comparative risk assessment of burden of disease and injury attributable to 67 risk factors and risk factor clusters in 21 regions, 1990-2010: a systematic analysis for the Global Burden of Disease Study 2010. Lancet. 2012;380:2224-2260.

2. Calhoun DA, Jones D, Textor S, et al. Resistant hypertension: diagnosis, evaluation, and treatment: a scientific statement from the American Heart Association Professional Education Committee of the Council for High Blood Pressure Research. Circulation. 2008;117:e510-e526.

3. Daugherty SL, Powers JD, Magid DJ, et al. Incidence and prognosis of resistant hypertension in hypertensive patients. Circulation. 2012;125:1635-1642.

4. Egan BM, Zhao Y, Axon RN, Brzezinski WA, Ferdinand KC. Uncontrolled and apparent treatment resistant hypertension in the United States, 1988 to 2008. Circulation. 2011;124:1046-1058. 
5. ALLHAT Officers and Coordinators for the ALLHAT Collaborative Research Group. The Antihypertensive and Lipid-Lowering Treatment to Prevent Heart Attack Trial. Major outcomes in high-risk hypertensive patients randomized to angiotensin-converting enzyme inhibitor or calcium channel blocker vs diuretic: the Antihypertensive and LipidLowering Treatment to Prevent Heart Attack Trial (ALLHAT). JAMA. 2002;288:2981-2997.

6. Roger VL, Go AS, Lloyd-Jones DM, et al. Heart disease and stroke statistics - 2012 update: a report from the American Heart Association. Circulation. 2012;125:e2-e220.

7. Jamerson K, Weber MA, Bakris GL, et al. Benazepril plus amlodipine or hydrochlorothiazide for hypertension in high-risk patients. $N$ Engl J Med. 2008;359:2417-2428.

8. de la Sierra A, Segura J, Banegas JR, et al. Clinical features of 8295 patients with resistant hypertension classified on the basis of ambulatory blood pressure monitoring. Hypertension. 2011;57: 898-902.

9. Chobanian AV, Bakris GL, Black HR, et al; National Heart, Lung, and Blood Institute Joint National Committee on Prevention, Detection, Evaluation, and Treatment of High Blood Pressure; National High Blood Pressure Education Program Coordinating Committee. The Seventh Report of the Joint National Committee on Prevention, Detection, Evaluation, and Treatment of High Blood Pressure (JNC 7). JAMA. 2003;289(19):2560-2572.

10. Marin JM, Carrizo SJ, Vicente E, Agusti AG. Long-term cardiovascular outcomes in men with obstructive sleep apnoea-hypopnoea with or without treatment with continuous positive airway pressure: an observational study. Lancet. 2005;365:1046-1053.

11. Young T, Palta M, Dempsey J, Skatrud J, Weber S, Badr S. The occurrence of sleep-disordered breathing among middle-aged adults. N Engl J Med. 1993;328:1230-1235.

12. Calhoun DA, Nishizaka MK, Zaman MA, Harding SM. Aldosterone excretion among subjects with resistant hypertension and symptoms of sleep apnea. Chest. 2004;125:112-117.

13. Logan AG, Perlikowski SM, Mente A, et al. High prevalence of unrecognized sleep apnoea in drug-resistant hypertension. $J$ Hypertens. 2001;19:2271-2277.

14. Somers VK, Dyken ME, Clary MP,Abboud FM. Sympathetic neural mechanisms in obstructive sleep apnea. J Clin Invest. 1995;96: 1897-1904.

15. Pratt-Ubunama MN, Nishizaka MK, Boedefeld RL, Cofield SS, Harding SM, Calhoun DA. Plasma aldosterone is related to severity of obstructive sleep apnea in subjects with resistant hypertension. Chest. 2007;131:453-459.

16. Friedman O, Bradley TD, Logan AG. Influence of lower body positive pressure on upper airway cross-sectional area in drug-resistant hypertension. Hypertension. 2013;61:240-245.

17. Ziegler MG, Mills PJ, Loredo JS, Ancoli-Israel S, Dimsdale JE. Effect of continuous positive airway pressure and placebo treatment on sympathetic nervous activity in patients with obstructive sleep apnea. Chest 2001;120:887-893.

18. Lozano L, Tovar JL, Sampol G, et al. Continuous positive airway pressure treatment in sleep apnea patients with resistant hypertension: a randomized, controlled trial. J Hypertens. 2010;28:2161-2168.

19. Gaddam K, Pimenta E, Thomas SJ, et al. Spironolactone reduces severity of obstructive sleep apnoea in patients with resistant hypertension: a preliminary report. J Hum Hypertens. 2010;24: 532-537.

20. Conn JW. Presidential address. I. Painting background. II. Primary aldosteronism, a new clinical syndrome. J Lab Clin Med. 1955;45:3-17.

21. Strauch B, Zelinka T, Hampf M, Bernhardt R, Widimsky J Jr. Prevalence of primary hyperaldosteronism in moderate to severe hypertension in the Central Europe region. J Hum Hypertens. 2003; 17: 349-352.

22. Born-Frontsberg E, Reincke M, Rump LC, et al. Cardiovascular and cerebrovascular comorbidities of hypokalemic and normokalemic primary aldosteronism: results of the German Conn's Registry. J Clin Endocrinol Metab. 2009;94:1125-1130.

23. Kaplan NM. Hypokalemia in the hypertensive patient, with observations on the incidence of primary aldosteronism. Ann Intern Med. 1967;66: 1079-1090.
24. Sinclair AM, Isles CG, Brown I, Cameron H, Murray GD, Robertson JW. Secondary hypertension in a blood pressure clinic. Arch Intern Med. 1987; 147:1289-1293.

25. Douma S, Petidis K, Doumas M, et al. Prevalence of primary hyperaldosteronism in resistant hypertension: a retrospective observational study. Lancet. 2008;371:1921-1926.

26. Calhoun DA, Nishizaka MK, Zaman MA, Thakkar RB, Weissmann P. Hyperaldosteronism among black and white subjects with resistant hypertension. Hypertension. 2002;40:892-896.

27. Gallay BJ, Ahmad S, Xu L, Toivola B, Davidson RC. Screening for primary aldosteronism without discontinuing hypertensive medications plasma aldosterone-renin ratio. Am J Kidney Dis. 2001;37:699-705.

28. Eide IK, Torjesen PA, Drolsum A, Babovic A, Lilledahl NP. Low-renin status in therapy-resistant hypertension: a clue to efficient treatment. J Hypertens. 2004;22:2217-2226.

29. Droyvold WB, Midthjell K, Nilsen TI, Holmen J. Change in body mass index and its impact on blood pressure: a prospective population study. Int J Obes (Lond). 2005;29:650-655.

30. Bentley-Lewis R, Adler GK, Perlstein T, et al. Body mass index predicts aldosterone production in normotensive adults on a high-salt diet. J Clin Endocrinol Metab. 2007;92:4472-4475.

31. Rossi GP, Belfiore A, Bernini G, et al. Body mass index predicts plasma aldosterone concentrations in overweight-obese primary hypertensive patients. J Clin Endocrinol Metab. 2008;93:2566-2571.

32. Tuck ML, Sowers J, Dornfeld L, Kledzik G, Maxwell M. The effect of weight reduction on blood pressure, plasma renin activity, and plasma aldosterone levels in obese patients. $N$ Engl J Med. 1981;304: 930-933.

33. Pantanetti P, Garrapa GG, Mantero F, Boscaro M, Faloia E, Venarucci D. Adipose tissue as an endocrine organ? A review of recent data related to cardiovascular complications of endocrine dysfunctions. Clin Exp Hypertens. 2004;26:387-398.

34. Ehrhart-Bornstein M, Lamounier-Zepter V, Schraven A, et al. Human adipocytes secrete mineralocorticoid-releasing factors. Proc Natl Acad Sci U S A. 2003;100:14211-14216.

35. Krug AW, Vleugels K, Schinner S, et al. Human adipocytes induce an ERK1/2 MAP kinases-mediated upregulation of steroidogenic acute regulatory protein (StAR) and an angiotensin II-sensitization in human adrenocortical cells. Int J Obes (Lond). 2007;31:1605-1616.

36. Mehta AN, Fenves A. Current opinions in renovascular hypertension. Proc (Bayl Univ Med Cent). 2010;23:246-249.

37. Safian RD, Textor SC. Renal-artery stenosis. N Engl J Med. 2001;344: 431-442.

38. Textor SC, Lerman L. Renovascular hypertension and ischemic nephropathy. Am J Hypertens. 2010;23:1159-1169.

39. Anderson GH Jr, Blakeman N, Streeten DH. The effect of age on prevalence of secondary forms of hypertension in 4429 consecutively referred patients. J Hypertens. 1994;12:609-615.

40. Crowley JJ, Santos RM, Peter RH, et al. Progression of renal artery stenosis in patients undergoing cardiac catheterization. Am Heart J. 1998;136:913-918.

41. Messerli FH, Bangalore S, Makani H, et al. Flash pulmonary oedema and bilateral renal artery stenosis: the Pickering syndrome. Eur Heart J. 2011;32:2231-2235.

42. Conlon PJ, Little MA, Pieper K, Mark DB. Severity of renal vascular disease predicts mortality in patients undergoing coronary angiography. Kidney Int. 2001;60:1490-1497.

43. Pickering TG, Herman L, Devereux RB, et al. Recurrent pulmonary oedema in hypertension due to bilateral renal artery stenosis: treatment by angioplasty or surgical revascularisation. Lancet. 1988;2:551-552.

44. Eklof H, Ahlstrom H, Magnusson A, et al. A prospective comparison of duplex ultrasonography, captopril renography, MRA, and CTA in assessing renal artery stenosis. Acta Radiol. 2006;47:764-774.

45. Murphy TP, Cooper CJ, Dworkin LD, et al. The Cardiovascular Outcomes with Renal Atherosclerotic Lesions (CORAL) study: rationale and methods. J Vasc Interv Radiol. 2005;16:1295-1300. 
46. Gray BH. Intervention for renal artery stenosis: endovascular and surgical roles. J Hypertens Suppl. 2005;23:S23-S29.

47. Mackrell PJ, Langan EM 3rd, Sullivan TM, et al. Management of renal artery stenosis: effects of a shift from surgical to percutaneous therapy on indications and outcomes. Ann Vasc Surg. 2003;17:54-59.

48. van Jaarsveld BC, Krijnen P, Pieterman H, et al. The effect of balloon angioplasty on hypertension in atherosclerotic renal-artery stenosis. Dutch Renal Artery Stenosis Intervention Cooperative Study Group. N Engl J Med. 2000;342:1007-1014.

49. Bax L, Woittiez AJ, Kouwenberg HJ, et al. Stent placement in patients with atherosclerotic renal artery stenosis and impaired renal function: a randomized trial. Ann Intern Med. 2009;150:840-848, W150-W151.

50. Wheatley K, Ives N, Gray R, et al. Revascularization versus medical therapy for renal-artery stenosis. N Engl J Med. 2009;361:1953-1962.

51. Weinberg MD, Olin JW. Stenting for atherosclerotic renal artery stenosis: one poorly designed trial after another. Cleve Clin J Med. 2010;77:164-171.

52. Chrysochou C, Kalra PA. Current management of atherosclerotic renovascular disease - what have we learned from ASTRAL? Nephron Clin Pract. 2010;115:c73-c81.

53. Hirsch AT, Haskal ZJ, Hertzer NR, et al. ACC/AHA Guidelines for the Management of Patients with Peripheral Arterial Disease (lower extremity, renal, mesenteric, and abdominal aortic): a collaborative report from the American Associations for Vascular Surgery/Society for Vascular Surgery, Society for Cardiovascular Angiography and Interventions, Society for Vascular Medicine and Biology, Society of Interventional Radiology, and the ACC/AHA Task Force on Practice Guidelines (writing committee to develop guidelines for the management of patients with peripheral arterial disease) - summary of recommendations. JVasc Interv Radiol. 2006;17:1383-1397, quiz 1398.

54. Chrysochou C, Foley RN, Young JF, Khavandi K, Cheung CM, Kalra PA. Dispelling the myth: the use of renin-angiotensin blockade in atheromatous renovascular disease. Nephrol Dial Transplant. 2012;27:1403-1409.

55. Plouin PF, Bax L. Diagnosis and treatment of renal artery stenosis. Nat Rev Nephrol. 2010;6:151-159.

56. Olin JW, Froehlich J, Gu X, et al. The United States Registry for Fibromuscular Dysplasia: results in the first 447 patients. Circulation. 2012;125:3182-3190

57. Pascual A, Bush HS, Copley JB. Renal fibromuscular dysplasia in elderly persons. Am J Kidney Dis. 2005;45:e63-e66.

58. Jayawardene S, Reidy J, Scoble J. Clinical picture: ipsilateral atherosclerotic and fibromuscular renal artery stenosis. Lancet. 2000;356:2138

59. Aqel R, Gupta R, Zoghbi G. Coexistent fibromuscular dysplasia and atherosclerotic renal artery stenosis. J Invasive Cardiol. 2005;17: 572-573.

60. Aqel R, AlJaroudi WW, Hage FG, Nanda NC. Renal artery fibromuscular dysplasia is a cause of refractory hypertension in the elderly. Echocardiography. 2009;26:109-110.

61. Turnbull JM. The rational clinical examination. Is listening for abdominal bruits useful in the evaluation of hypertension? JAMA. 1995;274:1299-1301.

62. Vollmer WM, Sacks FM, Ard J, et al. Effects of diet and sodium intake on blood pressure: subgroup analysis of the DASH sodium trial. Ann Intern Med. 2001;135:1019-1028.

63. Pimenta E, Stowasser M, Gordon RD, et al. Increased dietary sodium is related to severity of obstructive sleep apnea in patients with resistant hypertension and hyperaldosteronism. Chest. 2013;143:978-983.

64. Pimenta E, Gaddam KK, Oparil S, et al. Effects of dietary sodium reduction on blood pressure in subjects with resistant hypertension: results from a randomized trial. Hypertension. 2009;54: 475-481.

65. Appel LJ, Moore TJ, Obarzanek E, et al. A clinical trial of the effects of dietary patterns on blood pressure. DASH Collaborative Research Group. N Engl J Med. 1997;336:1117-1124.
66. Bramlage P, Pittrow D, Wittchen HU, et al. Hypertension in overweight and obese primary care patients is highly prevalent and poorly controlled. Am J Hypertens. 2004;17:904-910.

67. Aucott L, Poobalan A, Smith WC, Avenell A, Jung R, Broom J. Effects of weight loss in overweight/obese individuals and long-term hypertension outcomes: a systematic review. Hypertension. 2005;45: 1035-1041.

68. Whelton SP, Chin A, Xin X, He J. Effect of aerobic exercise on blood pressure: a meta-analysis of randomized, controlled trials. Ann Intern Med. 2002;136:493-503.

69. Kokkinos PF, Narayan P, Colleran JA, et al. Effects of regular exercise on blood pressure and left ventricular hypertrophy in African-American men with severe hypertension. $N$ Engl J Med. 1995;333:1462-1467.

70. Dimeo F, Pagonas N, Seibert F, Arndt R, Zidek W, Westhoff TH. Aerobic exercise reduces blood pressure in resistant hypertension. Hypertension. 2012;60:653-658.

71. Hanselin MR, Saseen JJ, Allen RR, Marrs JC, Nair KV. Description of antihypertensive use in patients with resistant hypertension prescribed four or more agents. Hypertension. 2011;58:1008-1013.

72. Daugherty SL, Powers JD, Magid DJ, et al. The association between medication adherence and treatment intensification with blood pressure control in resistant hypertension. Hypertension. 2012;60:303-309.

73. Vaclavik J, Sedlak R, Plachy M, et al. Addition of spironolactone in patients with resistant arterial hypertension (ASPIRANT): a randomized, doubleblind, placebo-controlled trial. Hypertension. 2011;57:1069-1075.

74. Garg JP, Elliott WJ, Folker A, Izhar M, Black HR. Resistant hypertension revisited: a comparison of two university-based cohorts. Am J Hypertens. 2005;18:619-626.

75. Taler SJ, Textor SC, Augustine JE. Resistant hypertension: comparing hemodynamic management to specialist care. Hypertension. 2002;39: 982-988.

76. Ernst ME, Carter BL, Goerdt CJ, et al. Comparative antihypertensive effects of hydrochlorothiazide and chlorthalidone on ambulatory and office blood pressure. Hypertension. 2006;47:352-358.

77. Sica DA. Chlorthalidone: has it always been the best thiazide-type diuretic? Hypertension. 2006;47:321-322.

78. Murray MD, Deer MM, Ferguson JA, et al. Open-label randomized trial of torsemide compared with furosemide therapy for patients with heart failure. Am J Med. 2001;111:513-520.

79. Nishizaka MK, Zaman MA, Calhoun DA. Efficacy of low-dose spironolactone in subjects with resistant hypertension. Am J Hypertens. 2003; 16:925-930.

80. Chapman N, Dobson J, Wilson S, et al. Effect of spironolactone on blood pressure in subjects with resistant hypertension. Hypertension. 2007;49:839-845.

81. Ouzan J, Perault C, Lincoff AM, Carre E, Mertes M. The role of spironolactone in the treatment of patients with refractory hypertension. Am J Hypertens. 2002;15:333-339.

82. Sever PS, Dahlof B, Poulter NR, et al. Prevention of coronary and stroke events with atorvastatin in hypertensive patients who have average or lower-than-average cholesterol concentrations, in the Anglo-Scandinavian Cardiac Outcomes Trial - Lipid Lowering Arm (ASCOT-LLA): a multicentre randomised controlled trial. Lancet. 2003;361:1149-1158.

83. de Souza F, Muxfeldt E, Fiszman R, Salles G. Efficacy of spironolactone therapy in patients with true resistant hypertension. Hypertension. 2010;55:147-152.

84. Gaddam K, Corros C, Pimenta E, et al. Rapid reversal of left ventricular hypertrophy and intracardiac volume overload in patients with resistant hypertension and hyperaldosteronism: a prospective clinical study. Hypertension. 2010;55:1137-1142.

85. Pitt B, Zannad F, Remme WJ, et al. The effect of spironolactone on morbidity and mortality in patients with severe heart failure. Randomized Aldactone Evaluation Study Investigators. $N$ Engl J Med. 1999;341: 709-717.

86. Pitt B, Remme W, Zannad F, et al. Eplerenone, a selective aldosterone blocker, in patients with left ventricular dysfunction after myocardial infarction. N Engl J Med. 2003;348:1309-1321. 
87. Calhoun DA, White WB. Effectiveness of the selective aldosterone blocker, eplerenone, in patients with resistant hypertension. J Am Soc Hypertens. 2008;2:462-468.

88. Saha C, Eckert GJ, Ambrosius WT, et al. Improvement in blood pressure with inhibition of the epithelial sodium channel in blacks with hypertension. Hypertension. 2005;46:481-487.

89. Mann JF, Schmieder RE, McQueen M, et al. Renal outcomes with telmisartan, ramipril, or both, in people at high vascular risk (the ONTARGET study): a multicentre, randomised, double-blind, controlled trial. Lancet. 2008;372:547-553.

90. Teo K, Yusuf S, Sleight P, et al. Rationale, design, and baseline characteristics of 2 large, simple, randomized trials evaluating telmisartan, ramipril, and their combination in high-risk patients: the Ongoing Telmisartan Alone and in Combination with Ramipril Global Endpoint Trial/Telmisartan Randomized Assessment Study in ACE Intolerant Subjects with Cardiovascular Disease (ONTARGET/TRANSCEND) trials. Am Heart J. 2004;148:52-61.

91. Guyenet PG. The sympathetic control of blood pressure. Nat Rev Neurosci. 2006; 7:335-346.

92. Scheffers IJ, Kroon AA, Tordoir JH, de Leeuw PW. Rheos Baroreflex Hypertension Therapy System to treat resistant hypertension. Expert Rev Med Devices. 2008;5:33-39.

93. Bisognano JD, Bakris G, Nadim MK, et al. Baroreflex activation therapy lowers blood pressure in patients with resistant hypertension: results from the double-blind, randomized, placebo-controlled rheos pivotal trial. J Am Coll Cardiol. 2011;58:765-773.
94. Bakris GL, Nadim MK, Haller H, Lovett EG, Schafer JE, Bisognano JD. Baroreflex activation therapy provides durable benefit in patients with resistant hypertension: results of long-term follow-up in the Rheos Pivotal Trial. J Am Soc Hypertens. 2012;6:152-158.

95. Krum H, Schlaich M, Whitbourn R, et al. Catheter-based renal sympathetic denervation for resistant hypertension: a multicentre safety and proof-of-principle cohort study. Lancet. 2009;373:1275-1281.

96. Esler MD, Krum H, Sobotka PA, Schlaich MP, Schmieder RE, Bohm M. Renal sympathetic denervation in patients with treatment-resistant hypertension (The Symplicity HTN-2 Trial): a randomised controlled trial. Lancet. 2010;376:1903-1909.

97. Schlaich MP, Sobotka PA, Krum H, Lambert E, Esler MD. Renal sympathetic-nerve ablation for uncontrolled hypertension. $N$ Engl J Med. 2009;361:932-934.

98. Esler MD, Krum H, Schlaich M, Schmieder RE, Bohm M, Sobotka PA. Renal sympathetic denervation for treatment of drugresistant hypertension: one-year results from the Symplicity HTN-2 randomized, controlled trial. Circulation. 2012;126:2976-2982.

99. Kandzari DE, Bhatt DL, Sobotka PA, et al. Catheter-based renal denervation for resistant hypertension: rationale and design of the SYMPLICITY HTN-3 Trial. Clin Cardiol. 2012;35:528-535.
Integrated Blood Pressure Control

\section{Publish your work in this journal}

Integrated Blood Pressure Control is an international, peer-reviewed open-access journal focusing on the integrated approach to managing hypertension and risk reduction. Treating the patient and comorbidities together with diet and lifestyle modification and optimizing healthcare resources through a multidisciplinary team approach constitute key

\section{Dovepress}

features of the journal. This journal is indexed on American Chemical Society's Chemical Abstracts Service (CAS). The manuscript management system is completely online and includes a very quick and fair peerreview system, which is all easy to use. Visit http://www.dovepress.com/ testimonials.php to read real quotes from published authors.

\footnotetext{
Submit your manuscript here: http://www.dovepress.com/integrated-blood-pressure-control-journal
} 